\title{
RESPUESTA DEL TOMATE A TIPOS DE ACOLCHADO PLÁSTICO Y NIVELES DE RIEGO CON CINTA
}

\section{TOMATO RESPONSE TO TYPES OF PLASTIC MULCH AND DRIP IRRIGATION LEVELS}

\author{
Marco A. Inzunza-Ibarra'*, Ernesto A. Catalán-Valencia', Magdalena \\ Villa-Castorena', Rutilo López-López ${ }^{2}$ y Ernesto Sifuentes-lbarra ${ }^{3}$
}

\begin{abstract}
'Centro de Investigación Disciplinaria en Relación Agua-Suelo-Planta-Atmósfera, Instituto Nacional de Investigaciones Forestales, Agrícolas y Pecuarias (INIFAP). Km. 6.5 Canal Sacramento margen derecho. 35140, Gómez Palacio, Dgo., México. Tel. (871) 159-0104. ²Campo experimental Huimanguillo, INIFAP. Carretera Huimanguillo - Cárdenas. 86400, Tabasco. ${ }^{3}$ Campo experimental Valle del Fuerte, CIRNO-INIFAP. Km 1609 Carretera México-Nogales, Juan José Ríos, Guasave, Sinaloa.
\end{abstract}

* Autor para correspondencia (inzunza.marco@inifap.gob.mx)

\section{RESUMEN}

El tomate (Solanum lycopersicum) es la hortaliza más popular en el mundo y en México. El estudio se realizó en el CENID-RASPA, INIFAP, en Gómez Palacio, Durango. El objetivo fue obtener la respuesta del cultivo del tomate al uso de distintos colores de cubierta plástica, criterios de riego y diferentes posiciones de cinta en el suelo. Se evaluó el rendimiento de fruto y la eficiencia de uso de agua (EUA) para el tomate variedad Yaqui. Se evaluaron 12 tratamientos provenientes del factorial combinatorio $3 \times 2 \times 2$, distribuidos en un diseño experimental completamente al azar. El primer factor, tipo de acolchado, se ensayó con tres niveles: negro, plata y sin acolchar, el segundo factor consistió de dos criterios de aplicación de agua, regar a 90 y a $100 \%$ de la evapotranspiración de referencia (ETo), y el tercer factor fue la profundidad de instalación de la cinta para riego, con dos niveles: a $25 \mathrm{~cm}$ y en la superficie del suelo. Los valores más altos de rendimiento de fruto, se mostraron en los tratamientos con acolchados y fueron superiores en $69.5 \%$, con respecto al tratamiento sin acolchar. El factor nivel de riego a $90 \%$ de ETo $(45 \mathrm{~cm})$ fue significativamente superior en $14.6 \%$ de rendimiento de fruto, en comparación al obtenido con el $100 \%$ de ETo $(51 \mathrm{~cm})$. En cambio, el rendimiento de fruto no fue estadísticamente diferente para la colocación de la cinta. En forma similar, para la variable eficiencia de uso de agua (EUA) se obtuvo un promedio de $7.03 \mathrm{~kg} \mathrm{~m}^{-3}$ con el uso del acolchado con películas plásticas y representó 70.2 $\%$, significativamente superior a la eficiencia obtenida con los tratamientos sin acolchar de $4.13 \mathrm{~kg} \mathrm{~m}^{-3}$. Asimismo, para el factor criterio de riego, la eficiencia de uso del agua del tomate, se incrementó en forma significativa $26.7 \%$ al irrigarse con el criterio de riego más seco (90\% ETo), comparado con la EUA de $5.35 \mathrm{~kg} \mathrm{~m}^{-3}$ obtenido al irrigar con $100 \%$ de la ETo.

Palabras clave: Solanum lycopersicum, cinta, uso eficiente del agua, trasplante, evapotranspiración de referencia.

\section{SUMMARY}

Tomato (Solanum lycopersicum) is the most popular vegetable in México and the world. This study was conducted at CENID-RASPA INIFAP, Gomez Palacio, Durango, and it evaluated the response of tomato to combinations of mulching type, irrigation criteria and depth of drip tape. Fruit yield and water use efficiency (EUA) of tomato cv. Yaqui were recorded. Twelve treatments resulting from a $3 \times 2 \times 2$ factorial arrangement were tested and distributed in a completely randomized experimental design. The first factor, type of mulching had three levels: black, silver and no mulch. The second factor consisted in two irrigation criteria: 90 and $100 \%$ of the reference evapotranspiration (ETo). The third factor, depth of the drip tape, included tape buried $25 \mathrm{~cm}$ deep and superficial tape. Highest fruit yield values were obtained on mulched treat- ments, and they were $69.5 \%$ higher than the treatment without mulching Treatments at $90 \%$ ETo $(45 \mathrm{~cm})$ yielded $14.6 \%$ higher than treatments at 100 $\%$ ETo $(51 \mathrm{~cm})$; the difference was significant. In contrast, fruit yield was not statistically different between drip tape depth. The effect of plastic mulching, measured as water use efficiency, showed that an average of $7.03 \mathrm{~kg} \mathrm{~m}^{-3}$ was obtained with plastic mulching compared to $4.13 \mathrm{~kg} \mathrm{~m}^{-3}$ for the treatment without mulching; this significant difference represented a $70.2 \%$ increase. Irrigation levels changed water use efficiency values significantly: under the drier criterion (90\% ETo) WUE increased by $26.7 \%$ irrigation at $100 \%$ ETo.

Index words: Solanum lycopersicum, drip tape, water use efficiency, transplant, reference evapotranspiration.

\section{INTRODUCCIÓN}

La aplicación del agua en forma eficiente es de los factores más importantes involucrados en la agricultura intensiva para el logro de la autosuficiencia alimentaria; sin embargo, el agua superficial y subterránea de buena calidad es un recurso cada vez más limitado debido a los impactos negativos causados por la urbanización, contaminación y cambio climático. Preservar en forma sustentable este recurso es trascendental y obliga a un manejo cada vez más eficiente del agua, especialmente en el sector agrícola por ser el mayor demandante de este vital líquido, al consumir alrededor de $70 \%$ del agua fresca disponible a nivel global (Li et al., 2015; Singh, 2014). Por lo anterior, existe la necesidad de incrementar la eficiencia de uso del agua (EUA) y generar tecnología para aprovechar otras fuentes de abastecimiento como podrían ser las aguas residuales o el agua de mar.

La utilización de métodos de riego de alta tecnología con el fin de proporcionar los requerimientos hídricos de los cultivos en forma óptima en su cantidad y oportunidad aunado a prácticas de manejo, como la utilización del acolchado con películas plásticas, han demostrado mejorar significativamente los sistemas productivos y por consecuencia un incremento significativo en la productividad 
del agua de riego, principalmente en cultivos hortícolas (Inzunza et al., 2007; López-López et al., 2015). Uno de estos cultivos, es sin duda el tomate (Solanum lycopersicum), cuya composición nutricional del fruto es afectada, en general por la variedad, año climático, condiciones de crecimiento, estado de madurez, luz, temperatura, suelo, fertilización y riego (Çetin y Uygan, 2008).

En este sentido, Patané y Cosentino (2010) dedujeron de estudios en diferentes localidades, que el tomate requiere de riego oportuno según la etapa del cultivo para obtener rendimientos económicamente viables en climas mediterráneos, y que al incrementar el déficit hídrico durante su etapa reproductiva tuvo una respuesta negativa en la producción y tamaño de fruto; sin embargo en el periodo vegetativo de emergencia a total floración, el rendimiento no disminuyó de manera significativa al someterse a déficit hídrico de 50 \% de la evapotranspiración de cultivo (ETC) comparado con aquel, que se irrigó al 100 \% de la ETc, afirman que esto representó 47 \% de ahorro de agua. Los autores coinciden que el agua es uno de los factores más importantes que benefician el crecimiento del fruto y su producción; por esto, la programación de los riegos es crucial para incrementar el rendimiento y la calidad del tomate (Wang et al., 2011). Además, resulta atractiva la explotación del tomate, por ser altamente remunerativo, si se utiliza tecnología adecuada, esto es, con riego continuo y de precisión en su aplicación como es el riego por goteo por cinta y con prácticas de manejo como el uso de acolchados plásticos (Panigrahi et al., 2014).

Patané et al. (2011) afirman que es posible incrementar la eficiencia de uso de agua en tomate y ahorrar volúmenes de este recurso hasta $46.6 \%$, al someter al cultivo a ligeros déficits hídricos, como reponer 50 \% de la evapotranspiración del cultivo (ETC), sin afectar significativamente su rendimiento e incrementar los parámetros de calidad del fruto, como el contenido de sólidos totales y el contenido de vitamina C. En forma similar, Favati et al. (2009) encontraron que el tomate optimiza su producción de fruto y alcanza altos contenidos nutricionales al aplicar el riego, al consumir $50 \mathrm{~mm}$ de la evapotranspiración del tomate (ETC) y restringir el riego en la etapa final de crecimiento un $50 \%$ de la ETc.

En este sentido, Hanson y May (2006) reportaron que el rendimiento del tomate es de $81.3 \mathrm{t} \mathrm{ha}^{-1}$ con una evapotranspiración de $648 \mathrm{~mm}$ y coeficientes de ajuste por desarrollo (kc) que fluctuaron de 0.95 a 1.09 durante la mitad del periodo vegetativo. Estudios de Wang et al. (2013) reportaron la importancia del manejo del riego y su interrelación con el incremento de la eficiencia de aprovechamiento del nitrógeno en tomate, al encontrar que el riego parcial alternado en su zona radical incrementa el fenómeno de difusión del flujo de nitratos en el suelo, lo que activa la demanda del nitrógeno por el cultivo.

Resulta significativamente importante generar información tendiente a incrementar la productividad del tomate por cultivarse en un área que fluctúa de 45 a 80 mil hectáreas en México y por debajo de su potencial productivo (Hernández-Leal et al., 2013; SIAP, 2015).

El presente estudio se desarrolló con el objetivo de evaluar la respuesta del cultivo del tomate, al uso de distintos colores de cubierta plástica, criterios de riego y diferentes posiciones de cinta en el suelo, con el fin de establecer alternativas de producción con alta eficiencia en el uso del agua y un incremento en su productividad.

\section{MATERIALES Y MÉTODOS}

El estudio se hizo en terrenos del campo experimental del Centro Nacional de Investigación Disciplinaria en la Relación Agua Suelo Planta Atmósfera (CENID RASPA) del Instituto Nacional de Investigaciones Forestales, Agrícolas y Pecuarias (INIFAP) ubicado en el ejido las Huertas, municipio de Gómez Palacio, Durango, en la Comarca Lagunera, localizado a $25^{\circ} 31^{\prime} 57^{\prime \prime} \mathrm{N}$ y $103^{\circ} 25^{\prime} 57^{\prime \prime} \mathrm{O}$, a una altitud de $1139 \mathrm{~m}$.

El clima de la Comarca Lagunera es de tipo desértico con bajo contenido de humedad atmosférica, precipitación pluvial promedio entre 200 y 300 mm anuales en la zona agrícola de riego y de 400 a $500 \mathrm{~mm}$ en la zona montañosa Oeste, con evaporación media anual de 2600 mm y temperatura media anual de $20^{\circ} \mathrm{C}$. Tanto el área de la llanura agrícola como gran parte de la zona montañosa presentan dos periodos bien definidos: el primero comprende siete meses de abril a octubre, en que la temperatura media mensual excede los $20^{\circ} \mathrm{C}$; el segundo abarca de noviembre a marzo, en el que la temperatura media mensual varía de 13.6 a $19.5^{\circ} \mathrm{C}$. Los meses más fríos son diciembre y enero, y en este último mes se registra el promedio más bajo de temperatura, de $5.8^{\circ} \mathrm{C}$ aproximadamente (Villa et al., 2005). Según García (2004), su clima se clasifica como BW (h') h w (e), que corresponde a muy árido, cálido con temperatura media anual de $22^{\circ} \mathrm{C}$ y temperatura media del mes más frío de $18^{\circ} \mathrm{C}$. El periodo libre de heladas es entre abril y octubre, y pueden presentarse granizadas en mayo. Las Iluvias son en verano, con un porcentaje de lluvias invernales que va de 5 a $10.2 \%$

Se utilizó un diseño experimental completamente al azar con tres repeticiones. Los factores en estudio fueron el acolchado plástico con tres niveles; color negro, plata y sin acolchar, el riego por goteo con dos niveles de aplicación (90 y 100 \% de la evapotranspiración de referencia 
ETo), estimada con la metodología de Hargreaves y Samani, recomendada para zonas áridas, y citada por Samani et al. (2011); y la profundidad de la cinta fue superficial y enterrada a $25 \mathrm{~cm}$. Se constituyó un factorial combinatorio $3 \times 2 \times 2$, con 12 tratamientos ensayados en campo (Cuadro 1). La unidad experimental consistió de tres líneas regantes de $10 \mathrm{~m}$ de longitud y $1.5 \mathrm{~m}$ de separación, la parcela útil fue determinada por la línea regante central, con dos hileras de plantas y se excluyeron los $2 \mathrm{~m}$ de los extremos para evitar el efecto de orilla.

El establecimiento del almácigo se realizó con 30 d de antelación para obtener plantas para el trasplante, el cual se realizó el 20 de julio del 2013, con semilla de tomate de la variedad Yaqui; se manejó una plantación a doble hilera, con una densidad de 40 mil plantas ha-1. Se utilizó riego por goteo con cinta tipo T-TAPE con espesor de pared de 0.38 $\mathrm{mm}$, calibre de $12.7 \mathrm{~mm}$ y un gasto de $2.20 \mathrm{~L} \mathrm{~h}^{-1}$ por metro lineal de cinta a una presión de operación de $41.4 \mathrm{KPa}$. La mitad de las líneas regantes en la parcela quedaron superficiales y la otra mitad se enterró a una profundidad de 25 $\mathrm{cm}$, la colocación del plástico se realizó de forma mecánica por medio de la denominada "acolchadora tipo INIFAP", el ancho del plástico fue de $1.20 \mathrm{~m}$, fue enterrado un total de $20 \mathrm{~cm}$ de cada lado, el espesor de la lámina plástica fue de $0.15 \mathrm{~mm}$ calibre 90. Para la formación del bulbo húmedo se regó un total de $33 \mathrm{~h}$ a una presión de $34.5 \mathrm{KPa}$ para evitar encharcamiento, esto con el fin de producir franjas de suelo humedecidas de 40 a $45 \mathrm{~cm}$ de ancho por línea regante, para el cálculo de la lámina de riego a aplicar se utilizó 90 y 100 \% de la evapotranspiración de referencia (ETo), respectivamente. Las láminas de riego totales fueron de 46 y $51 \mathrm{~cm}$ para cada caso y se consideró la formación del bulbo de mojado, el área de influencia de mojado de las líneas regantes, el gasto de la cinta y el porcentaje de la ETo.

Para la fertilización, en concordancia con el análisis de suelo (Cuadro 2), se utilizó la fórmula 120N-52P-26K, aplicada en 8 fracciones a través del ciclo del cultivo, disuelta en el agua de riego. Se utilizaron urea, nitrato de amonio, ácido fosfórico al $75 \%$ y nitrato de potasio como fuentes de nitrógeno, fósforo y potasio, respectivamente.
Durante el desarrollo del experimento se registraron las temperaturas del suelo con geotermómetros a $15 \mathrm{~cm}$ de profundidad del suelo. Fue colocada una unidad referida para cada tratamiento bajo estudio. Los sensores se conectaron a una estación de almacenamiento de información, para esto se utilizó un equipo tipo Campbell® Scientific con un datalogger modelo CR-10X (Barcelona, España). El equipo se programó para registrar el dato de la temperatura del suelo en forma continua cada 15 min y almacenar en la memoria del mismo.

Las plagas que se presentaron fueron mosquita blanca (Bemisia tabaci) y gusano del fruto (Heliothis virescens) y para su control se aplicó Confidor ${ }^{\circledR}$ (Imidacloprid) y Lannate ${ }^{\circledR}$ (Metomil), respectivamente. Para el control de hongos y enfermedades se utilizó Bayletón (Triadimefon). La cosecha se llevó a cabo manualmente durante 22 cortes. Las variables respuesta medidas fueron el rendimiento de fruto $\left(\mathrm{t} \mathrm{ha}^{-1}\right)$ y la eficiencia de uso del agua $\left(\mathrm{kg} \mathrm{m}^{-3}\right)$ del tomate. También se registró la temperatura del suelo por tratamiento $\left({ }^{\circ} \mathrm{C}\right)$ como variable auxiliar que cuantifique y explique la modificación del régimen térmico en los tratamientos con acolchado. La información fue analizada estadísticamente mediante análisis de varianza y comparación de medias (Tukey, 0.05) para lo cual se utilizó el programa estadístico SAS, (SAS Institute, 2008).

\section{RESULTADOS Y DISCUSIÓN}

De acuerdo con el análisis de varianza para rendimiento de fruto del tomate, que se muestra en el Cuadro 3, se observó alta significancia $(P \leq 0.01)$ para el factor tipos de acolchado (A), significancia para el factor criterio de riego (R) y no significancia para colocación de la cinta (CC). La interacción tipos de acolchado con criterios de riego ( $\mathrm{A} \times$ R) presentó significancia estadística para rendimiento. El análisis no mostró significancia para el resto de las interacciones dobles correspondientes al color de acolchado con colocación de la cinta $(\mathrm{A} \times \mathrm{CC})$ y la de criterios de riego con colocación de la cinta $(\mathrm{R} \times \mathrm{CC})$. Por último, el análisis no mostró significancia estadística para la interacción triple.

Para la eficiencia de uso del agua del tomate $\left(\mathrm{kg} \mathrm{m}^{-3}\right)$ se realizó el análisis de varianza (Cuadro 3); se encontraron

Cuadro 1. Características químicas del suelo del sitio experimental.

\begin{tabular}{lccccccc}
\hline Prof. $(\mathrm{cm})$ & $\mathrm{NO}_{3}\left(\mathrm{mg} \mathrm{kg}^{-1}\right)$ & $\mathrm{P}\left(\mathrm{mg} \mathrm{kg}^{-1}\right)$ & $\mathrm{K}\left(\mathrm{mg} \mathrm{kg}^{-1}\right)$ & $\mathrm{CO}_{3}\left(\mathrm{~g} \mathrm{~kg}^{-1}\right)$ & $\mathrm{CEe}\left(\mathrm{dS} \mathrm{m}^{-1}\right)$ & $\mathrm{CIC}\left(\mathrm{cmol} \mathrm{kg}^{-1}\right)$ & $\mathrm{pH}$ \\
\hline $0-30$ & 23.18 & 9.20 & 1045.83 & 53.0 & 0.79 & 24.4 & 8.47 \\
$30-60$ & 37.27 & 8.23 & 912.50 & 68.0 & 1.07 & 23.5 & 8.30 \\
$60-90$ & 43.42 & 9.67 & 833.30 & 86.6 & 1.03 & 23.4 & 8.15 \\
\hline
\end{tabular}

Prof.: profundidad de la muestra del suelo; $\mathrm{NO}_{3}$ : nitrato disponible; P. fósforo disponible; $\mathrm{K}$ : potasio disponible; $\mathrm{CO}_{3}$ : carbonatos totales; $\mathrm{CEe}$ : conductividad eléctrica; CIC: capacidad de intercambio catiónico. 
diferencias altamente significativas para A y $\mathrm{R}$; sin embargo, no se encontraron diferencias significativas para CC. Para el caso de la interacción $A \times R$, ésta fue altamente significativa, en cambio, no se encontró significancia estadística para el resto de las interacciones dobles ni para la triple interacción.

En el Cuadro 4 se muestra el análisis de comparación de medias de Tukey (0.05), del efecto combinado de los factores significativos con el promedio del factor no significativo. Se deduce que la mejor combinación, por su mayor rendimiento de fruto y eficiencia de uso del agua, fue el uso del acolchado color plata y aplicar $46 \mathrm{~cm}$ de agua durante el ciclo de desarrollo del tomate. El análisis indicó que esta combinación fue significativamente más alta y diferente al resto, lo que representó un incremento del 33 y $37.3 \%$ de rendimiento de fruto y eficiencia de uso del agua, respectivamente, con respecto al valor próximo más alto. Al compararse la citada combinación con los tratamientos que no usaron películas plásticas y que se regaron con el nivel de riego alto $(51 \mathrm{~cm})$, se obtuvo un incremento productivo de fruto y de eficiencia de uso de agua de 109 y $132 \%$, respectivamente. Estos incrementos en la producción son causados por las condiciones favorables para el crecimiento de la planta, propiciados por las películas plásticas, como son la conservación de la humedad al reducir la evaporación directa del suelo, mayor eficiencia en la obtención de los nutrimentos al tener mayor disponibilidad por dichas condiciones (Inzunza-Ibarra et al., 2010; Li et al., 2015).
Es importante mencionar que a pesar de que el criterio de riego del 90 \% de ETo, significó una lámina de agua aplicada menor $(46 \mathrm{~cm})$ comparada con la del criterio de riego al $100 \%$ de la ETo con $51 \mathrm{~cm}$, se obtuvo un rendimiento y eficiencia de uso de agua por el tomate, significativamente mayor, al asociarse con el efecto del plástico. Esto pudo deberse a la mayor conservación y disponibilidad de agua por el acolchado plástico y por otro lado en los tratamientos húmedos, a las condiciones anaeróbicas prolongadas en la zona radical o lixiviación de nutrientes por el exceso de humedad fuera de la influencia radical, que provocó la reducción del rendimiento. Hechos similares concuerdan con los citados por Hernández-Leal et al. (2013), que afirman que el exceso de riego en etapas tempranas provocó un mayor crecimiento vegetativo con disminución de la floración.

Debido a la no significancia del factor colocación de la cinta (Cuadro 3), los valores medios del rendimiento de fruto y eficiencia de uso del agua resultaron ser estadísticamente iguales. La cinta enterrada a $25 \mathrm{~cm}$, tuvo un efecto insignificante, por que únicamente incrementó $1.29 \%$ del rendimiento de fruto, con respecto al obtenido con la cinta colocada superficialmente. Esto pudo deberse a que con la cinta enterrada se disminuyeron las pérdidas por evaporación y se mantuvo una mayor disponibilidad de humedad en la zona radical. Deducciones similares se reportan por Machado y Oliveira (2005), que concluyen que para el cultivo de tomate la cinta a diferentes colocaciones de profundidad en el suelo no afecta el rendimiento del fruto.

\section{Cuadro 2. Tratamientos ensayados en campo para el tomate en el CENID-RASPA INIFAP.}

\begin{tabular}{lccc}
\hline Tratamiento & Tipo de acolchado & Nivel de riego $(\%$ ETo) & Colocación de la cinta \\
\hline N1S & Negro & 90 & $0 \mathrm{~cm}$ \\
N1E & Negro & 90 & $25 \mathrm{~cm}$ \\
N2S & Negro & 100 & $0 \mathrm{~cm}$ \\
N2E & Negro & 100 & $25 \mathrm{~cm}$ \\
P1S & Plata & 90 & $0 \mathrm{~cm}$ \\
P1E & Plata & 90 & $25 \mathrm{~cm}$ \\
P2S & Plata & 100 & $0 \mathrm{~cm}$ \\
P2E & Plata & 100 & $25 \mathrm{~cm}$ \\
SA1S & Sin Acolchar & 90 & $0 \mathrm{~cm}$ \\
SA1E & Sin Acolchar & 90 & $25 \mathrm{~cm}$ \\
SA2S & Sin Acolchar & 100 & $0 \mathrm{~cm}$ \\
SA2E & Sin Acolchar & 100 & $25 \mathrm{~cm}$ \\
\hline
\end{tabular}

ETo: evapotranspiración del referencia; N1S: con acolchado negro, irrigada con 90 \% ETo y cinta colocada a 0 cm; N1E: acolchado negro, 90 \% ETo y cinta a $25 \mathrm{~cm}$ de la superficie del suelo; N2S: con acolchado negro, irrigada con $100 \%$ ETo y cinta colocada a $0 \mathrm{~cm}$; N2E: con acolchado negro, irrigada con $100 \%$ ETo y cinta colocada a $25 \mathrm{~cm}$; P1S : acolchado color plata, $90 \%$ ETo y cinta a 0 cm; P1E : acolchado color plata, $90 \%$ ETo y cinta a 25 cm; P2S: acolchado color plata, $100 \%$ ETo y cinta a 0 cm; P2E: acolchado plata, irrigado al $100 \%$ ETo, y cinta a $25 \mathrm{~cm}$; SA1S: sin acolchar, al $90 \%$ ETo y cinta a 0 cm; SA1E: sin acolchar, al 90 \% ETo y cinta a 25 cm; SA2S: sin acolchar, al $100 \%$ ETo y cinta a 0 cm; SA2E: sin acolchar, al 100 $\%$ ETo y cinta a $25 \mathrm{~cm}$. 
Cuadro 3. Cuadrados medios del análisis de varianza y prueba de $\mathrm{F}$ para rendimiento de fruto $(\mathrm{Y})$ y eficiencia de uso del agua (EUA) del tomate.

\begin{tabular}{|c|c|c|c|}
\hline Fuente de variación & gl & Rendimiento (t ha-1) & EUA $\left(\mathrm{kg} \mathrm{m}^{-3}\right)$ \\
\hline Tipo de acolchado(A) & 2 & $818.4 \star \star$ & $36.33 * *$ \\
\hline Nivel de riego ( $R$ ) & 1 & $136.7 *$ & $18.40 * *$ \\
\hline Colocación cinta (CC) & 1 & 1.2 & 0.076 \\
\hline$A \times R$ & 2 & $213.7 \star \star$ & $10.53 * *$ \\
\hline$A \times C C$ & 2 & 15.0 & 0.55 \\
\hline $\mathrm{R} \times \mathrm{CC}$ & 1 & 26.4 & 1.16 \\
\hline$A \times R \times C C$ & 2 & 56.5 & 2.33 \\
\hline Error & 24 & 25.1 & 1.02 \\
\hline
\end{tabular}

*: $P \leq 0.05, * *: P \leq 0.01 ;$ gl: grados de libertad; EUA: eficiencia de uso del agua.

Cuadro 4. Efecto combinado del tipo de acolchado sobre el rendimiento ( $\left.\mathrm{t} \mathrm{ha}^{-1}\right)$ y eficiencia de uso del agua del tomate $\left(\mathrm{kg} \mathrm{m}^{-3}\right)$.

\begin{tabular}{lll}
\hline Combinación & Rendimiento $\left(\mathrm{t} \mathrm{ha}^{-1}\right)$ & Eficiencia de uso del agua $\left(\mathrm{kg} \mathrm{m}^{-3}\right)$ \\
\hline P1 & $42.8 \mathrm{a}^{\dagger}$ & $9.31 \mathrm{a}$ \\
P2 & $29.2 \mathrm{~b}$ & $5.72 \mathrm{~b}$ \\
$\mathrm{~N} 1$ & $31.1 \mathrm{~b}$ & $6.77 \mathrm{~b}$ \\
$\mathrm{~N} 2$ & $32.2 \mathrm{~b}$ & $6.32 \mathrm{~b}$ \\
SA1 & $19.6 \mathrm{c}$ & $4.26 \mathrm{c}$ \\
SA2 & $20.5 \mathrm{c}$ & $4.01 \mathrm{c}$ \\
\hline
\end{tabular}

${ }^{\top}$ Medias con letras iguales no son estadísticamente diferentes (Tukey, 0.05); P1: tratamiento con acolchado color plata irrigado con el 90 ETo; P2: acolchado plata irrigado con el 100 \% ETo; N1: acolchado negro irrigado con el $90 \%$ ETo; N2: acolchado negro irrigado con el $100 \%$ ETo; SA1: sin acolchar irrigado con 90 \% ETo; SA2: sin acolchar irrigado con el 100 \% ETo.

Al analizar las interacciones con mayor detalle, se deduce que el efecto de la película plástica en las variables de respuesta del tomate, fue significativamente más alta que sin acolchar, para los dos niveles de riego en estudio (Cuadro 5). Para el rendimiento de fruto se manifestaron incrementos significativos de 7.3 y de 10.2 t ha-1 $^{-1} 88$ y 50 $\%)$ en los tratamientos acolchados con respecto a aquellos sin acolchar, al aplicarse una lámina de riego de 46 y $56 \mathrm{~cm}$, respectivamente.

El rendimiento del tomate tuvo un incremento del $38 \%$ al utilizar el acolchado color plata con respecto al acolchado color negro regados ambos con láminas de riego de $46 \mathrm{~cm}$. Bajo este nivel de riego el tratamiento con plástico color plata, superó al tratamiento sin acolchar $118 \%$. Estas diferencias no fueron tan acentuadas cuando se regó con láminas de $56 \mathrm{~cm}$ por que las producciones de tomate fueron estadísticamente iguales entre colores de acolchado, pero significativamente más altas, un $50 \%$, comparada con la obtenida sin acolchar.

El acolchado incrementó significativamente la productividad del agua del cultivo al ser comparada con los tratamientos que se desarrollaron sin película plástica (Cuadro
5). El tratamiento con acolchado color plata y que se regó con $90 \%$ de la ETo $(46 \mathrm{~cm})$, incrementó significativamente $37 \%$ el EUA del cultivo, con respecto a la obtenida con la película plástica negra bajo la misma condición de humedad. Esta diferencia fue más contundente $(116 \%)$ al comparar el acolchado color plata con la obtenida con el tratamiento sin acolchar, bajo el mismo nivel de riego (46 $\mathrm{cm}$ ). Resultados similares fueron reportados por Hou et al. (2010) en papa (Solanum tuberosum) en el noroeste de China. El color del acolchado no tuvo influencia significativa en los valores de eficiencia del agua para el nivel alto de riego (100 ETo o aplicación de $56 \mathrm{~cm}$ de agua) porque el acolchado negro incrementó solo 12 \% la productividad del agua con respecto al de color plata; sin embargo, para esta condición hídrica el usar el acolchado incrementó significativamente, en promedio 51 \%, el valor de la eficiencia de uso del agua del tomate comparado con el tratamiento sin cubierta plástica (Cuadro 5).

\section{Temperatura del suelo}

Independientemente de los tratamientos ensayados, la temperatura media diaria del suelo a los $15 \mathrm{~cm}$ de profundidad disminuyó de 26 a $20^{\circ} \mathrm{C}$ durante el periodo de 
Cuadro 5. Medias de rendimiento de fruto $\left(\mathrm{t} \mathrm{ha}^{-1}\right)$ y de eficiencia de uso del agua $\left(\mathrm{kg} \mathrm{m}^{-3}\right)$ del tomate, resultantes de la interacción entre tipos de acolchado y niveles de riego.

\begin{tabular}{lcccc}
\hline Tipo acolchado & $90 \mathrm{ETo}\left(\mathrm{t} \mathrm{ha}^{-1}\right)$ & $100 \mathrm{ETo}\left(\mathrm{t} \mathrm{ha}^{-1}\right)$ & $90 \mathrm{ETo}\left(\mathrm{kg} \mathrm{m}^{-3}\right)$ & $100 \mathrm{ETo}\left(\mathrm{kg} \mathrm{m}^{-3}\right)$ \\
\hline Negro & $31.1 \mathrm{~b}$ & $32.2 \mathrm{a}$ & $6.8 \mathrm{~b}$ & $6.4 \mathrm{a}$ \\
Plata & $42.8 \mathrm{a}$ & $29.2 \mathrm{a}$ & $9.3 \mathrm{a}$ & $5.7 \mathrm{a}$ \\
Sin acolchar & $19.6 \mathrm{c}$ & $20.5 \mathrm{~b}$ & $4.3 \mathrm{c}$ & $4.0 \mathrm{~b}$ \\
\hline
\end{tabular}

ETo: evapotranspiración de referencia.

medición de esta variable. Esto reflejó el enfriamiento progresivo del ambiente debido a la transición estacional verano-otoño ocurrida dentro de los últimos 68 d del ciclo del cultivo (1 sept -7 nov), cuando la temperatura media del aire disminuyó de 28.8 a $19.4^{\circ} \mathrm{C}$. Las amplitudes de las oscilaciones diarias de la temperatura del suelo delimitadas por las temperaturas máximas y mínimas, registradas durante el periodo de medición, fueron afectadas de distinta manera por los tratamientos, destacándose el acolchado plástico sobre los otros dos factores estudiados (Figura 1).

Las dosis de riego y los criterios de colocación de la cinta afectaron de manera moderada a la temperatura del suelo, principalmente a la temperatura máxima diaria promedio. La mayor dosis de riego disminuyó $0.6{ }^{\circ} \mathrm{C}$ el valor de esta variable en relación con la dosis de riego más baja. Por su parte, la cinta superficial disminuyó $0.7^{\circ} \mathrm{C}$ la temperatura máxima con respecto a la cinta enterrada. El acolchado plástico tuvo un efecto importante en la temperatura del suelo, especialmente sobre la temperatura mínima diaria (Figura 2). El acolchado negro incrementó $1.2^{\circ} \mathrm{C}$ la temperatura mínima diaria promedio, y redujo $43 \%$ la amplitud de la temperatura del suelo diaria promedio en relación con el tratamiento sin acolchar. Esto se debió a que el color negro del acolchado absorbe más de $90 \%$ de la radiación y lo transforma en calor. El incremento de la temperatura del suelo por el acolchado varía con el clima y la época del año o estación, ya que se han reportado incrementos de temperatura mayores de $3{ }^{\circ} \mathrm{C}$ como los consignados en primavera y verano por Singh y Kamal (2012) para cubierta plástica negra.

El acolchado color plata incrementó $1.6^{\circ} \mathrm{C}$ la temperatura mínima diaria promedio en relación con el tratamiento sin acolchar. Este valor, ligeramente superior al producido por el acolchado negro se contrapone con lo esperado, ya que estudios previos han reportado que el acolchado plata refleja hasta $39 \%$ de la radiación de onda corta incidente, por lo cual debería calentar menos el suelo que el acolchado negro (Gough, 2001; Rajablariani et al., 2012). Factores como el sombreado del acolchado por el cultivo y su bloqueo por polvo, así como cierto nivel de trasmisión de la radiación por el acolchado color plata podrían explicar esta situación.
El aumento de la temperatura mínima del suelo producido por el acolchado plástico contribuyó de manera importante al incremento del rendimiento de fruto $70 \%$ en promedio, observado con los acolchados color plata y negro; valor comparable al $63 \%$ de incremento de fruto de tomate producido por el acolchado color plata y reportado por Cetin y Uygan (2008). La contribución del acolchado al rendimiento fue confirmada mediante un análisis de correlación del rendimiento promedio por tratamiento con los correspondientes valores promedio de temperatura máxima y mínima del suelo, de lo que resultaron correlaciones de 0.11 para las temperaturas máximas y 0.52 para las temperaturas mínimas.

La temperatura de la zona radical es importante porque afecta procesos fisiológicos como la absorción de agua y nutrientes. También es importante para la supervivencia de las plantas porque las raíces tienen una menor temperatura óptima y son más sensibles a valores extremos de temperatura que el follaje (Ham et al., 1993). En el presente estudio, debido a la época del año en que fue realizado, los valores promedio de temperatura máxima correspondientes a los tratamientos con y sin acolchado fueron similares y menores que $25{ }^{\circ} \mathrm{C}$, valor máximo de temperatura del suelo ensayado por Paszt et al. (2014) con el cual lograron el máximo rendimiento de tomate. Esto refuerza la idea de la contribución importante que el aumento de la temperatura mínima del suelo tuvo en el rendimiento del cultivo.

\section{CONCLUSIONES}

Los tratamientos bajo acolchado incrementaron significativamente el rendimiento, $44.9 \%$ con el color plata y $33.3 \%$ con el color negro comparado con el tratamiento sin acolchado. El acolchado de color plata no presentó diferencias significativas con respecto al de color negro, por lo que el uso de un determinado color lo determinará entre otros factores, el precio de los mismos. El acolchado plástico propició condiciones de humedad favorables en el suelo al obtener $52 \%$ más de fruto al irrigarse sólo con $46 \mathrm{~cm}$ de agua en comparación con los tratamientos sin acolchar y aplicaciones de $56 \mathrm{~cm}$. Para el tomate no se recomienda colocar la cinta enterrada puesto que no incrementa significativamente el rendimiento de fruto. 


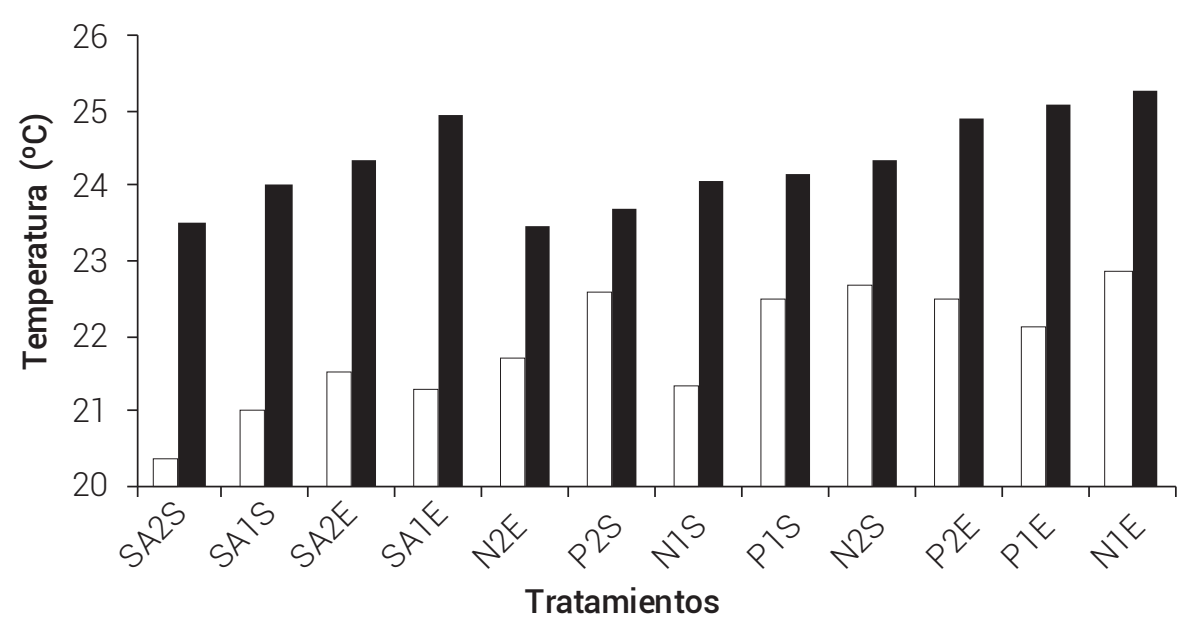

Figura 1. Efecto de los tratamientos sobre la temperatura del suelo. SA: sin acolchar; N: acolchado color negro; P. acolchado color plata; 1: criterio de riego al $90 \%$ de la ETo; 2: Criterio de riego al $100 \%$ de la ETo; S: cinta de riego colocada en la superficie del suelo; E: cinta de riego colocada a $25 \mathrm{~cm}$ de la superficie del suelo.

\section{-T máxima diaria promedio $\quad \square$ T mínima diaria promedio}

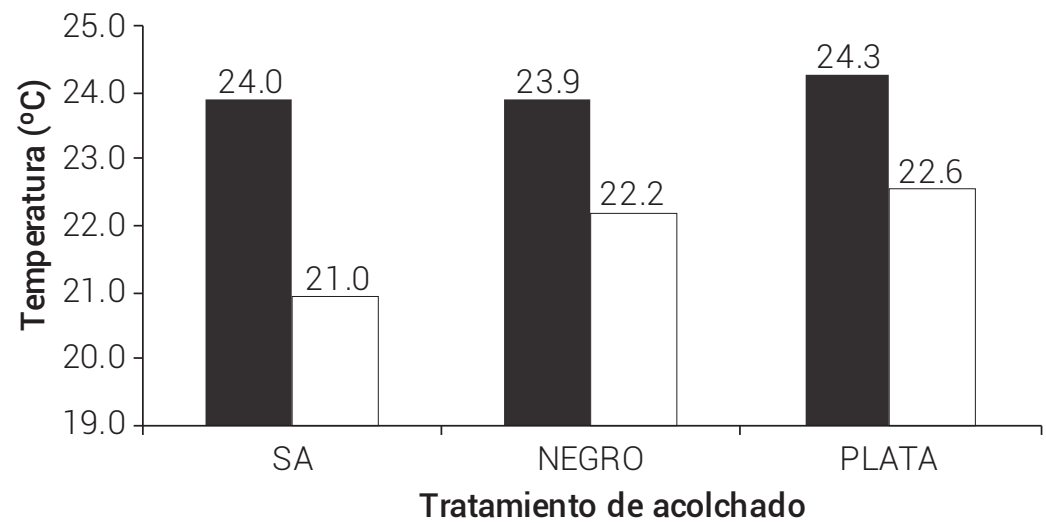

Figura 2. Efecto del acolchado plástico en la temperatura del suelo. SA: sin acolchar.

Los mejores tratamientos fueron los que utilizaron el acolchado de color plata o negro en combinación con cinta superficial para el tomate ya que este sistema aumentó la producción y redujo el volumen de agua requerido.

\section{BIBLIOGRAFÍA}

Çetin Ö. and D. Uygan (2008) The effect of drip line spacing, irrigation regimes and planting geometries of tomato on yield, irrigation water use efficiency and net return. Agricultural Water Management 95:949-958.

Favati F., S. Lovelli, F. Galgano, V. Miccolis, T. Di Tommaso and V. Candido (2009) Processing tomato quality as affected by irrigation scheduling. Scientia Horticulturae 122:562-571.

García E. (2004) Modificaciones al Sistema de Clasificación Climática de Köppen. Serie Libros. Núm. 6. Instituto de Geografía, UNAM. México, D.F. 90 p.

Gough R. E. (2001) Color of plastic mulch affects lateral root develop- ment but not root system architecture in pepper. HortScience 36:66-68.

Ham J. M., G. J. Kluitenberg and W. J. Lamont (1993) Optical properties of plastic mulches affect the field temperature regime. Journal of the American Society for Horticultural Science 118:188-193.

Hanson B. R. and D. M. May (2006) Crop evapotranspiration of processing tomato in the San Joaquin Valley of California, USA. Irrigation Science 24:211-221.

Hou X. Y., F. X. Wang, J. J. Han, S. Z. Kang and S. Y. Feng (2010) Duration of plastic mulch for potato growth under drip irrigation in an arid region of Northwest China. Agricultural and Forest Meteorology 150:115-121.

Hernández-Leal E., R. Lobato-Ortiz, J. J. García-Zavala, D. Reyes-López, A. Méndez-López, 0. Bonilla-Barrientos y A. Hernández-Bautista (2013) Comportamiento agronómico de poblaciones $\mathrm{F}_{2}$ de híbridos de tomate (Solanum lycopersicum L.). Revista Fitotecnia Mexicana 36:209-215.

Inzunza I. M. A., S. F. Mendoza M., E. A. Catalán V., M. M. Villa C., I. Sánchez C. y A. Román L. (2007) Productividad del chile jalapeño en condiciones de riego por goteo y acolchado plástico. Revista Fitotecnia 
Mexicana 30:429-436.

Inzunza-Ibarra M. A., M. Villa-Castorena, E. A. Catalán-Valencia y A. RománLópez (2010) Extracción de nutrientes y producción de chile jalapeño bajo acolchado plástico y niveles de riego. Terra Latinoamericana 28:211-218.

Li X., H. Shi, J. Šimůnek, X. Gong and Z. Peng (2015) Modeling soil water dynamics in a drip-irrigated intercropping field under plastic mulch. Irrigation Science 33:289-302.

López-López R., M. A. Inzunza-Ibarra, I. Sánchez-Cohen, A. Fierro-Álvarez and E. Sifuentes-Ibarra (2015) Water use efficiency and productivity of habanero pepper (Capsicum chinense Jacq.) based on two transplanting dates. Water Science \& Technology 71:885-891.

Machado R. M. A. and M. R. G. Oliveira (2005) Tomato root distribution, yield and fruit quality under different subsurface drip irrigation regimes and depths. Irrigation Science 24:15-24.

Panigrahi P., R. K. Sharma, M. Hasan and S. S. Parihar (2014) Deficit irrigation scheduling and yield prediction of 'Kinnow' mandarin (Citrus reticulate Blanco) in a semiarid region. Agricultural Water Management 140:48-60

Paszt L. S., P. Trzciński, M. Bakalarska, R. Holownicki, P. Konopacki and W. Treder (2014) The influence of heated soil in crop of "Tamaris" tomato plants on the biological activity of the rhizosphere soil. Advances in Microbiology 4:191-201.

Patané C. and S. L. Cosentino (2010) Effects of soil water deficit on yield and quality of processing tomato under a Mediterranean climate. Agricultural Water Management 97:131-138.

Patané C., S. Tringali and 0 . Sortino (2011) Effects of deficit irrigation on biomass, yield, water productivity and fruit quality of processing tomato under semi-arid Mediterranean climate conditions. Scientia Horticulturae 129:590-596.
Rajablariani H. R., F. Hassankhan and R. Rafezi (2012) Effect of colored plastic mulches on yield of tomato and weed biomass. International Journal of Environmental Science and Development 3:590-593.

Samani Z., S. Bawazir, R. Skaggs, J. Longworth, A. Piñon and V. Tran (2011) A simple irrigation scheduling approach for pecans. Agricultural Water Management 98:661-664.

SAS Institute (2008) (SAS/STAT) 2008. Ver. 9.2 SAS Institute Inc. Cary, USA. 429 p.

SIAP, Sistema de Información Agroalimentaria y Pesquera (2015) Anuario estadístico de la producción agrícola. Sistema de Información Agroalimentaria y Pesquera, SAGARPA, México. http://www. gob.mx/cms/uploads/attachment/file/92738/Cuadros_tabulares_2014.compressed.pdf (Diciembre 2015).

Singh A. K. and S. Kamal (2012) Effect of black plastic mulch on soil temperature and tomato yield in mid hills of Garhwal Himalayas. Journal of Horticulture and Forestry 4:78-80.

Singh A. K. (2014) Simulation-optimization modeling for conjunctive water use management. Agricultural Water Management 141:23-29.

Villa C. M. M., E. A. Catalán V. y M. A. Inzunza I. (2005) Análisis de información climática para usos agrícolas. Agrofaz 5:717-724.

Wang F., S. Kang, T. Du, F. Li and R. Qiu (2011) Determination of comprehensive quality index for tomato and its response to different irrigation treatments. Agricultural Water Management 98:12281238.

Wang Y., F. Liu, L. S. Jensen, A. de Neergaard and C. R. Jensen (2013) Alternate partial root-zone irrigation improves fertilizer-N use efficiency in tomatoes. Irrigation Science 31:589-598. 